\title{
Enabling oral amphotericin B delivery by merging the benefits of prodrug approach and nanocarrier-mediated drug delivery
}

\author{
Kaushik Thanki ${ }^{1}$, Tushar Date ${ }^{1}$, Sanyog Jain*
}

Centre for Pharmaceutical Nanotechnology, Department of Pharmaceutics, National Institute of Pharmaceutical Education and Research (NIPER), S.A.S. Nagar (Mohali), Sector 67, Punjab - 160062, India

*Corresponding author: Dr. Sanyog Jain

Email: sanyogjain@ niper.ac.in, sanyogjain@ rediffmail.com

Tel: +91172-2292055, Fax: +91172-2214692

Table S1: Statistical treatment of the various dependent variables over design space

\begin{tabular}{|l|c|l|c|c|}
\hline Particulars & Droplet size & PDI & \% Transmittance & Drug loading \\
\hline Model p value & 0.0004 & $<0.0001$ & 0.0003 & $<0.0001$ \\
\hline $\mathrm{R}^{2}$ & 0.9404 & 0.9189 & 0.9111 & 0.9170 \\
\hline Adj $\mathrm{R}^{2}$ & 0.8979 & 0.8919 & 0.8666 & 0.9004 \\
\hline Pred R & 0.8243 & 0.8633 & 0.7204 & 0.8528 \\
\hline Adeq precision & 14.604 & 17.206 & 13.742 & 21.256 \\
\hline \multicolumn{5}{|c|}{ Adj: Adjusted; Pred: Predicted; Adeq: Adequate }
\end{tabular}

